\title{
Enseigner la physique en milieu difficile: co-construction de la référence en classe dédoublée et en classe entière
}

\section{Patrice Venturini et Chantal Amade-Escot}

Dans le but de mieux comprendre les pratiques ordinaires, cet article compare la manière dont une enseignante de physique co-construit avec les élèves la référence dans une classe dédoublée et une classe entière, en zone d'éducation prioritaire. Il montre qu'en classe dédoublée il est possible d'établir un partenariat effectif avec les élèves pour faire avancer le savoir, sous certaines conditions. Il montre aussi que les stratégies mises en cuvre sont beaucoup moins opérantes en classe entière, la collaboration étant beaucoup plus aléatoire, ce qui amène l'enseignante à adopter une posture en «surplomb».

L'étude présentée concerne la comparaison des stratégies d'enseignement de la physique en classe dédoublée et en classe entière d'une professeure expérimentée exerçant dans un établissement situé en milieu difficile dans une zone d'éducation prioritaire (ZEP). Plus particulièrement, la contribution analyse la coconstruction de la référence dans la classe. Après avoir rappelé quelques constats récurrents relatifs aux pratiques d'enseignement et d'apprentissage en ZEP, nous précisons la problématique que nous venons d'ébaucher. Celle-ci nous conduit à exposer brièvement le modèle de l'action didactique conjointe qui est le cadre théorique dans lequel elle s'inscrit, ainsi que la méthodologie qui y est rattachée. Nous discutons ensuite, à la lumière de quelques extraits de séances, les modalités de construction de la référence propres à chacun des deux contextes analysés.

\section{Enseigner en Zone d'Education Prioritaire}

Nous donnons ici quelques points de repère liés au contenu de notre recherche, issus pour une grande partie de la revue de question effectuée sur ce thème par Rochex et Kherroubi (2004).

Selon les résultats qu'ils rapportent, les enseignants de ces milieux difficiles ont à gérer plusieurs contradictions, entre: 
- une logique d'apprentissage et les logiques de socialisation et d'éducation, ces dernières étant souvent considérées comme préalables et extérieures aux activités disciplinaires, et donc concurrentes (Rochex \& Kherroubi, 2004);

- une logique collective et une logique individuelle, dans la conduite de la classe. Si l'individualisation des activités permet une progression différenciée et évite à l'enseignant les phases collectives difficiles à gérer sur le plan disciplinaire (Peltier-Barbier, 2004), elle empêche cependant la construction de connaissances communes à l'ensemble de la classe. A l'opposé, le travail collectif facilite l'introduction des nouveaux savoirs le plus souvent à partir d'ostensions ou par une sorte de maïeutique basée sur les propos glanés dans la classe. Toutefois, il ne permet pas pour autant leur appropriation par les élèves, en tout cas en termes d'apprentissage qu'il ne resterait qu'à systématiser (Butlen, Barbier-Peltier \& Pezard, 2002);

- une logique d'apprentissage et une logique d'engagement dans l'apprentissage, favorisée par la réussite immédiate dans la tâche proposée. Si la réussite renforce la dynamique d'apprentissage et facilite de plus les relations dans la classe, elle amène aussi les enseignants à adapter les objectifs et les stratégies au niveau supposé des élèves. Les tâches proposées sont simplifiées et le plus souvent fermées. Les savoirs ainsi mis à l'étude relèvent de compétences de bas-niveau, le travail est étroitement piloté par les consignes de l'enseignant, l'oral y est privilégié (Bautier \& Goigoux, 2004; Peltier-Barbier, 2004; Van Zanten, 2001). Ce sur-ajustement didactique aux caractéristiques des élèves réduit l'investissement nécessaire pour apprendre ainsi que les apprentissages réalisés, avec des effets cumulatifs importants dans le temps.

De plus, la centration sur la réussite immédiate conduit les élèves à se focaliser sur les tâches elles-mêmes et sur leur réalisation, d'autant que le travail exigé est le plus souvent limité au temps de l'activité en classe. Ils sont donc principalement dans l'action, et plus sensibles aux traits de surface des situations éducatives qu'aux savoirs qu'elles permettent d'institutionnaliser (Peltier-Barbier, 2004).

Pour conclure, la gestion de ces dilemmes et contradictions par les ensei-gnants s'opère généralement aux dépens d'une «ressaisie» distanciée des tâches permettant l'identification des liens qui les unissent ainsi qu'une compréhension de leurs enjeux réels. Elle empêche le travail (extérieur à la tâche elle-même) nécessaire pour l'appropriation des savoirs en faisant obstacle à la prise en compte des objets scolaires comme «un monde d'objets à interroger, sur lesquels ils [les élèves] peuvent (et ils doivent) exercer des activités de pensée et un travail spécifique», dans une "attitude de secondarisation» conditionnant la réussite scolaire (Bautier \& Goigoux, 2004, p. 91). 


\section{Problémat ique}

L'ensemble des constats précédents tend à montrer que les logiques d'action des élèves et des enseignants en milieu difficile se renforcent l'une l'autre et conduisent à un fonctionnement didactique caractérisé par des situations de faible épaisseur épistémologique. Le souci de maintenir une relation didactique, d'enrôler les élèves dans la tâche, aurait pour conséquence une baisse des exigences sur les savoirs enseignés. Ces processus, qui engagent de manière conjointe enseignants et élèves, témoignent de tensions et de conflits d'intentions susceptibles de conduire aux difficultés rencontrées à la fois par les enseignants et les élèves dans ces milieux (Monnier \& Amade-Escot, 2009). Cette analyse rejoint d'une certaine manière les conclusions des recherches menées dans des classes plus ordinaires, selon lesquelles l'avancée du processus d'enseignement-apprentissage procède d'ajustements plus ou moins maîtrisés, plus ou moins adaptés quand surviennent des dysfonctionnements même légers contraignant l'ensei-gnant à (ré)agir en situation (Amade-Escot, 2002). A cet égard, si l'on considère avec Rochex (1997) que les milieux difficiles donnent à voir comme à travers une loupe, des phénomènes didactiques plus génériques, on comprend l'intérêt d'examiner les pratiques d'enseignement ordinaires qui y sont menées.

Toutefois, on imagine mal, même en milieu difficile, des pratiques monolithiques et il est probable que la résolution dans l'action des contradictions précédemment signalées s'opère de manière variable selon les contextes, selon par exemple la nature des savoirs en jeu ou selon les conditions dans lesquelles ils sont transmis. C'est cette seconde hypothèse que nous avons choisie d'examiner dans une perspective comparatiste dans le cadre de cet article (Joshua, 2002; Mercier, Schubauer-Leoni \& Sensevy, 2002). L'enseignement de la physique au collège offre cette possibilité parce qu'il est possible de le mener tantôt en classe dédoublée, tantôt en classe entière, sans que cette alternance ne conduise à des types d'activité fortement différenciés. En effet, dans un cas comme dans l'autre, les enseignants privilégient une forme de «TP - cours» dans la mesure où les programmes officiels recommandent de partir de situations problématiques que les élèves doivent résoudre en mettant en œuvre une démarche d'investigation ${ }^{1}$ (MEN, 2007). Cependant la taille différente des groupes permet d'envisager une organisation et un déroulement différent des activités, en faisant par exemple plus de place à la composante expérimentale en classe dédoublée, et permet d'offrir ainsi des contextes différents mais comparables. Compte-tenu des observations précédemment rapportées sur les ZEP, l'apport d'une étude comparatiste nous a semblé davantage fondé dans le cas d'un enseignant affichant clairement sa volonté de transmettre les savoirs disciplinaires tels que les programmes les définissent et demandent de les transmettre.

En résumé, cette étude a pour but de comparer des formes d'enseignement-apprentissage de la physique en classe dédoublée et en classe entière, telles qu'elles ont été menées en ZEP au collège, par un enseignant déclarant vouloir trans- 
mettre les contenus disciplinaires selon les recommandations du programme. L'objectif est de profiter de l'effet «loupe» lié au milieu difficile pour produire des connaissances sur les processus facilitant ou non l'engagement des élèves dans l'étude et l'attitude de secondarisation à partir de l'analyse de quelques modalités remarquables de co-construction de la référence en classe de physique.

Nous intéressant aux pratiques d'enseignement ordinaires en milieu difficile, et plus particulièrement aux logiques d'action des élèves et des enseignants, nous nous sommes inscrits dans le cadre de théorie de l'action didactique conjointe professeur-élève(s) (Sensevy \& Mercier, 2007; Schubauer-Leoni \& Leutenegger, 2005).

\section{Construction de la référence dans l'action conjointe professeur-élève(s)}

Si la question de la référence et donc celle d'une légitimité extérieure des savoirs enseignés en classe a été formalisée dès 1985 par Chevallard (1991), elle continue à faire débat. Nous l'envisageons ici à partir d'une analyse ascendante de la transposition didactique (Schubauer-Leoni \& Leutenegger, 2005), prenant en compte à la fois son aspect externe, «celui des pratiques de références» qui servent de repères au travail didactique ${ }^{2}$, et son aspect interne, "hic et nunc, qui renvoie au procès des transactions, au jeu didactique en tant que tel et à ses enjeux» (Sensevy \& Mercier, 2007, p. 196). Suivant ces auteurs, nous considérons la référence de manière holiste, en envisageant sa construction comme le résultat d'un cheminement dialectique sur un continuum reliant ses caractéristiques interne et externe. C'est pourquoi l'investigation des phénomènes de transposition didactique ne peut se situer "ni [que] dans les savoirs, ni [que] dans les sujets - enseignants et apprenants - mais dans leur action conjointe. Il s'agit ... de considérer ce travail de coproduction de connaissances à la lumière des pratiques culturelles qui le légitiment» (Schubauer-Leoni \& Leutenegger, 2005, p. 408).

L'enjeu de la problématique transpositive considérée dans cette perspective relève d'une co-construction des savoirs (Schubauer-Leoni, 2008). En effet, le processus d'enseignement-apprentissage nécessite la construction en classe d'une référence ${ }^{3}$ qui tienne lieu de "point de repère pour que les gestes de l'enseignant et de l'élève puissent se coordonner et produire une histoire didactique» (p. 72). Cette référence n'est pas pour autant commune à l'élève et au professeur, qui ont des buts distincts et des rapports différents aux savoirs en jeu. Mais elle rend possible une certaine interdépendance de leurs buts permettant ainsi l'avancée du savoir. Construire la référence, c'est donc construire «un lieu de médiation constitué d'un 'système d'objets ${ }^{4}$ ' pour enseigner et pour apprendre» (p. 75), c'est-à-dire générer un milieu pour l'enseignement et l'apprentissage (Brousseau, 1986, 1990; Chevallard, 1992), 
Ce milieu dont on a pu montrer qu'il articule des dimensions institutionnelles et situationnelles 5 (Amade-Escot $\&$ Venturini, 2009), évolue dynamiquement dans le temps au fur et à mesure que les savoirs avancent dans la classe sous l'effet de l'action conjointe de l'enseignant et de l'élève. Soulignons que dans l'action conjointe, ce processus évolutif, appelé mésogenèse ${ }^{6}$, est associé à la manière dont sont gérés «les territoires et des temporalités» (Schubauer-Leoni, 2008, p. 76), c'est-à-dire d'une part le partage des responsabilités entre ensei-gnant et élève dans l'avancée du savoir (topogénèèse ${ }^{7}$ ) et d'autre part l'avancée du temps didactique (chronogénèse).

Pour ce qui concerne cette étude, nous nous attacherons plus particulièrement à décrire les processus mésogénétiques à l'œuvre dans deux situations contrastées, classe entière et classe dédoublée, pour analyser l'influence du contexte sur la co-construction de la référence. Celle-ci en tant que résultat du cheminement dialectique entre ses caractéristiques interne et externe, concernera ce qui se dit ou se fait dans le cours des transactions didactiques, convoquant suivant les moments:

- des éléments institutionnels: savoirs (notions, concepts...), usages (expériences canoniques...), etc.

- des éléments émergeant en situation: idées momentanément partagées dans la classe, produits des institutionnalisations successives, etc.

Cet en effet en considérant tous ces éléments que nous rendons compte du processus d'élaboration d'une référence toujours en construction dans la classe. Pour cela, nous avons mis en œuvre la méthodologie suivante.

\section{Méthodologie}

\section{Le cadre général}

La recherche a été réalisée dans la classe d'un collège de banlieue d'une grande métropole régionale, considéré comme un des plus difficiles de l'agglomération comportant une vingtaine de classes. Aux dires de l'enseignante, le renouvellement des enseignants et des élèves est important. Les agressions physiques entre élèves sont régulières, les agressions verbales en classe et les vols fréquents, la violence est quotidienne. L'enseignante que nous avons observée a 10 ans d'ancienneté dont neuf passés dans diverses ZEP de l'hexagone, elle est très attachée à son métier et malgré les conditions difficiles, l'exerce toujours avec plaisir. Elle dit être bien installée dans la classe de septième année de scolarité obligatoire (5e du collège français) qui a servi de support à l'étude. Cette classe comporte 17 élèves, dont 10 filles et 5 garçons présents lors des observations. Elle est décrite par l'enseignante comme ayant "une bonne dynamique» (entretien ante). En effet, même si la gestion de cette classe reste un problème de tous les instants, la participation orale aux activités et aux expériences proposées est importante. Toutefois, les élèves ont de nombreuses difficultés, principalement vis-à-vis de 
l'écrit, d'autant qu'ils "travaillent très peu à la maison» (entretien ante). Dix d'entre eux sont d'ailleurs «en échec» aux dires de l'enseignante qui rapporte en ces termes le bilan du conseil de classe (entretien ante).

\section{Le recueil des données}

Nous nous sommes fortement inspirés de la méthodologie proposée par Leutenegger (2003). Le protocole de recueil de données croise, en les articulant, des informations issues de corpus relatifs aux différents éléments de la triade réunissant l'enseignant, l'enseigné et l'objet d'enseignement-apprentissage. Dans cette perspective, nous avons recueilli différents types de données relatifs:

- à l'activité de l'enseignante en classe. Nous avons filmé deux séances consécutives de physique menées dans la même salle, en classe dédoublée (8 élèves dont les 5 garçons) puis en classe entière, portant sur le thème «Sources de lumière, objets diffusants, condition de visibilité d'un objet». Celui-ci, qui débute l'optique en cinquième (MEN, 2007), traite de l'existence de deux types de sources, primaires et secondaires, respectivement caractérisées par l'émission et la diffusion de la lumière, ainsi que de la vision, conditionnée par la pénétration de la lumière dans l'œil;

- aux documents distribués aux élèves (fiches à compléter à propos des différentes étapes du cours - expérience, observation, interprétation et conclusions - et exercice d'application);

- aux enjeux didactiques, en termes de savoirs et de démarches, repérés au regard des programmes actuels du collège;

- aux objectifs et aux analyses de l'enseignante à propos des séances observées. Des entretiens ante- et post-séances ont été réalisés avec elle pour cerner a priori ses objectifs et obtenir une première analyse à chaud de son activité;

- aux acquisitions des élèves en classe dédoublée. Nous avons demandé à cinq élèves désignés par l'enseignante comme représentatifs de la classe en termes de résultats scolaires en physique, de reformuler oralement au cours d'un entretien collectif, ce qu'ils avaient fait et appris au cours de la séance effectuée en début de matinée.

\section{Le traitement des données}

Les vidéos constituent le corpus principal. Leur visionnement a permis de construire le synopsis de chaque séance (Leutenegger, 2003) mettant en relation un repérage temporel, les différentes tâches et leurs visées, les ressources disponibles pour leur réalisation. Pour chacune des tâches, nous avons décrit les modalités de travail et détaillé ce que disent et font enseignant et élèves. Les tableaux 1 et 2 présentent une synthèse des synopsis de séances. 


\section{Tableau 1: Synopsis condensé de la séance en classe dédoublée (en gris l'épisode analysé)}

\section{Temps}

(minutes)

$1-2$

$6-12$

Classer 4 sources (lampe, soleil, neige, balle de ping-pong) selon

$$
\begin{aligned}
& \text { leurs propriétés } \\
& \text { Débat collectif }
\end{aligned}
$$

Voit-on une balle blanche dans l'obscurité?

Expérience de pensée (prévisions) sur la vision dans l'obscurité d'une lampe, d'une balle blanche, de cette balle éclairée en lumière blanche puis en lumière rouge.

Réalisation effective de l'expérience par l'enseignante. Formalisation individuelle des observations sur les fiches. Contrôle oral des réponses.

Institutionnalisation dictée des observations.

$$
\text { Enjeux de savoir }
$$

Cerner le nouveau domaine d'étude qu'est l'optique

Sources primaires émettant de la lumière et sources secondaires

Visibilité d'une source secondaire seulement si elle est éclairée par une source primaire

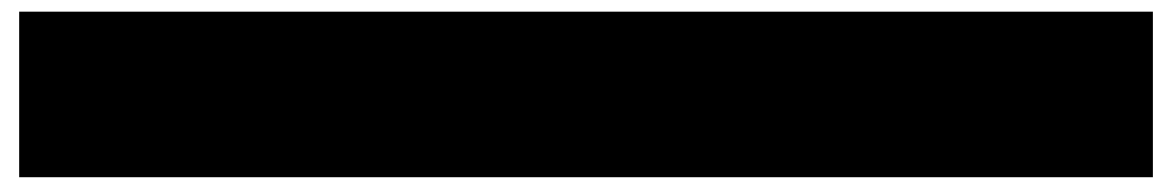

$35-48$
Classification de différentes sources de lumière

Exercices individuels

Correction orale.

Institutionnalisation dictée

Qui voit le bateau sur la mer?

(Utilisation d'une bande dessinée)

Exercice individuel
Réinvestissement des propriétés des sources primaires et secondaires

Préparer la réflexion sur les conditions de visibilité d'un objet (avoir les yeux ouverts et regarder l'objet)

\section{Tableau 2: Synopsis condensé de la séance en classe entière (en gris l'épisode analy-}

\section{Temps (minutes)}

Nature et organisation des activités

Qui voit le bateau sur la mer?

(Utilisation d'une bande dessinée)

Correction orale de l'exercice réalisé à la fin de la séance

en classe dédoublée.

Institutionnalisation dictée
Enjeux de savoir

Préparer la réflexion sur les conditions de visibilité d'un objet (avoir les yeux ouverts et regarder l'objet) 


\begin{tabular}{|c|c|c|}
\hline $\begin{array}{l}\text { Temps } \\
\text { (minutes) }\end{array}$ & Nature et organisation des activités & Enjeux de savoir \\
\hline $23-36$ & $\begin{array}{l}\text { Biographie d'Al-Azhem } 9 \\
\text { Activité documentaire: lecture d'un texte historique, } \\
\text { questions à faire la maison }\end{array}$ & $\begin{array}{l}\text { Repères historiques en lien avec } \\
\text { la culture scientifique arabe, } \\
\text { non exploités dans cette séance }\end{array}$ \\
\hline $35-36$ & $\begin{array}{l}\text { L'oeil source primaire ou secondaire? } \\
\text { Débat collectif autour d'une question d'élève }\end{array}$ & L'œil, source secondaire \\
\hline
\end{tabular}

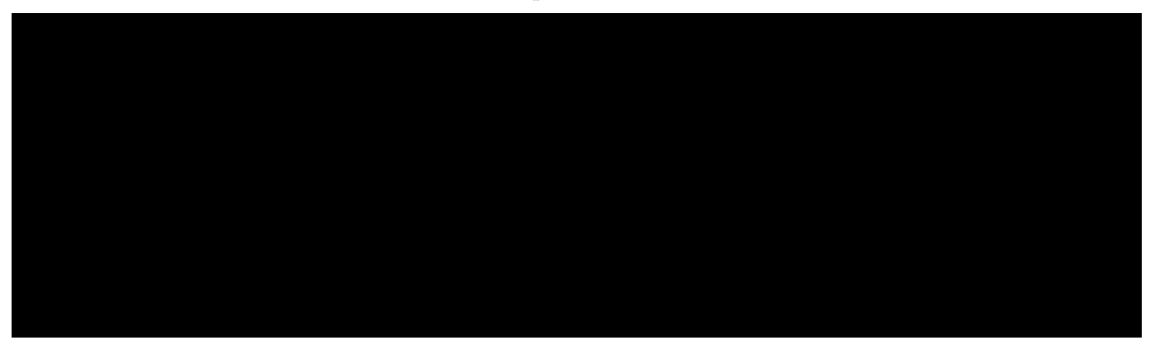

sé).

Cette première étape d'analyse fait apparaître un certain nombre d'épisodes, liés à des enjeux didactiques différents, structurant les séances. Parmi eux, sans nous interdire de faire référence à d'autres parties, nous avons retenu pour cet article deux épisodes portant sur des situations problématiques:

- La première («Pourquoi voit-on une balle de ping-pong si elle ne produit pas de lumière ?», question posée dans la fiche élève) est traitée en classe dédoublée (min 21-34, tableau 1) et concerne la distinction des propriétés des sources primaires (qui produisent de la lumière) et des sources secondaires, (qui diffusent une partie de la lumière qu'elles reçoivent).

- La seconde («Pourquoi voit-on les objets autour de nous alors que souvent ils ne sont pas directement éclairés par une source primaire ?») est travaillée en classe entière (min 37-49, tableau 2), et porte sur le rôle dans le phénomène des objets diffusants et directement éclairés.

Outre le fait que ces deux épisodes sont tous deux situés à la fin des deux séances, ils ont aussi été sélectionnés parce que leurs enjeux de savoirs sont emboités: le premier (en classe dédoublée) vise à cerner le phénomène de diffusion par les sources secondaires, le second (en classe entière) l'utilise pour interpréter des observations quotidiennes. Tous deux contribuent (avec d'autres) à modéliser la vision d'un objet par l'œil (au sens de la physique).

Pour interpréter les données vidéo, d'autres éléments ont été convoqués. Nous avons analysé des points de vue épistémologique et didactique les programmes officiels relatifs à l'optique en cinquième au collège (grade 7) ainsi que les documents d'accompagnement proposés par le ministère français. Cette analyse nous a permis d'identifier les enjeux de savoirs et la manière de les traiter, tels qu'ils ont été fixés par l'institution, ainsi que les difficultés qu'ils recèlent, 
afin d'éclairer avec ces éléments les situations observées. De la retranscription verbatim des entretiens ante- et post-séances, nous avons extrait les propos qui donnent du sens, selon nous, à certains évènements repérés lors du traitement des observations vidéo. Enfin, le contenu des fiches distribuées en physique et la démarche qu'elles induisent ont été examinés au regard des programmes.

\section{Résultats}

L'ensemble de ces analyses a permis de comparer les différentes situations examinées en nous intéressant plus particulièrement à la façon dont, au fil des interactions, professeur et élèves participent à l'élaboration du milieu pour l'étude (Johsua \& Félix, 2002). Compte tenu de la taille de l'article, nous avons choisi d'exemplifier à chaque fois cette comparaison à partir des traits les plus saillants de l'activité des enseignants articulée à celle des élèves.

\section{Enjeux didactiques}

Situons d'abord pour le lecteur les enjeux didactiques des deux épisodes analysés ici qui participent à la construction progressive d'un modèle scientifique de la vision et qui sont tout à fait conformes aux programmes officiels ${ }^{10}$. En effet, selon les deux conceptions très largement dominantes à cet âge (par exemple Andersson \& Karrqvist, 1983; Selley, 1996; Tiberghien, 1983), la lumière n'intervient que pour constituer un «bain" général dans lequel trempe l'objet, ou bien alors, elle éclaire l'objet, ce qui permet, dans l'un ou l'autre des cas, de le voir. Dans ces deux modèles spontanés, la lumière ne se propage pas jusqu'à l'œil. Le modèle du physicien, qui lui met en jeu la diffusion par l'objet éclairé de tout ou partie du rayonnement reçu, notamment en direction de l'œil, est pratiquement inexis-tant. De plus, la propriété d'émettre de la lumière est clairement et seulement attribuée à quelques objets (lampes, feu, soleil...), les sources secondaires (objets diffusants) en étant dépourvues. Les enjeux didactiques de cette séance, à savoir les conditions assurant la visibilité d'un objet (émission et diffusion de la lumière, respectivement par les sources primaires et secondaires puis réception par l'œil) correspondent donc des obstacles significatifs dans la construction des connaissances en optique. Leur caractère fondamental explique d'ailleurs qu' elles sont mises à l'étude à différents niveaux du cursus de physique (classe de 5 e, grade 7 , et classe de $1^{\mathrm{e}}$, grade 11 ).

\section{Stratégie utilisée par l'enseignante en classe entière et dédoublée}

La stratégie choisie par l'enseignante pour développer son enseignement peut se résumer en trois points, que nous allons développer tour à tour: respecter les exigences du programme officiel tant sur les contenus que sur la démarche à mettre en œuvre, utiliser une démarche de type maïeutique construite sur des échanges 
collectifs pour faire avancer le savoir, et faciliter le raisonnement des élèves en délimitant le milieu et en pointant les «traits pertinents» de ce dernier (Sensevy, Mercier \& Schubauer-Leoni, 2000; Schubauer-Leoni, 2008).

\section{Respecter les exigences du programme en termes de démarche et de contenu}

La structuration des deux épisodes analysés ici fait apparaître l'ambition de l'enseignante à l'égard des savoirs disciplinaires, ambition clairement affirmée lors de l'entretien ante. Leur organisation est pratiquement identique, agencée autour des principales étapes de la démarche d'investigation préconisée par les programmes (MEN, 2007), comme on peut le constater dans les tableaux 1 et 2: situation problème, réflexion collective sous forme de débat tant pour s'approprier la question et préparer l'expérience que pour discuter des observations expérimentales et de leur interprétation, phase d'institutionnalisation, opérationnalisation du modèle dans d'autres situations. Si les fiches distribuées récapitulent les questions et les expériences, elles laissent à la charge de l'élève la formalisation des observations, de leur interprétation et des conclusions à tirer (dans ce cas il s'agit toutefois d'un texte à compléter). Ce travail individuel prépare à chaque fois une discussion collective, suivie d'une institutionnalisation dictée, toujours relative aux savoirs exigibles des programmes. Le positionnement opéré par l'enseignante est donc résolument centré sur l'appropriation disciplinaire, selon les modalités proposées dans les programmes.

\section{Utiliser une démarche de type mä̈eutique basée sur des échanges collectifs}

Pour le rendre effectif, l'enseignante s'appuie beaucoup sur des échanges collectifs donnant lieu à une maïeutique classique comme en témoigne l'extrait 1 ciaprès. On peut y voir comment elle cherche, en interrogeant systématiquement les élèves, à les faire s'exprimer sur ce qu'ils savent pour faire progresser le savoir.

\section{Extrait 1: Exemple de démarche maïeutique (classe dédoublée, minute 22 à 23).}

P (Professeur): La lumière part de la lampe, elle va dans toute la salle ... elle va en particulier sur

Ra: $\quad$ sur la balle...

Si: $\quad$ elle tape sur la balle.

P: $\quad$ Elle tape euh...

Ho: $\quad$ La lumière, elle envoie des trucs sur la balle

P: $\quad$ Alors, la lampe envoie de la lumière à la balle. Après?

Ho: Et après, nous on la voit

P: $\quad$ Comment ça se fait?

Ho: $\quad$ Parce que. Parce que. La lampe envoie de la lumière à la balle, voilà, et après nous on peut la voir.

P: $\quad$ Comment ça se fait?

Ra: Grâce à la lumière aussi

Ho: Il y a quelque chose dans nos yeux 
Ka: $\quad$ Elle l'éclaire parce que la lumière est reçue du bon côté, voilà. Cette démarche privilégie largement l'oral à l'écrit, conformément aux stratégies mises en place par cette enseignante dans cette classe difficile: «je souhaite qu'il y ait toujours ce passage à l'écrit, mais il y en a beaucoup moins [que ce que je faisais en collège classique» (entretien ante). Elle s'appuie aussi sur une technique mésogénétique particulière que nous décrivons dans la section suivante et qui consiste à délimiter au fur et à mesure, dans le milieu didactique, quels sont les éléments à prendre en considération pour produire le raisonnement escompté. Cette façon de faire oriente son action qui vise à transmettre ce qu'elle considère essentiel pour ces élèves-là.

\section{Faciliter le raisonnement des élèves en délimitant les éléments à débattre dans le milieu et en pointant les traits pertinents}

Si l'on considère que le milieu construit pour l'étude comporte, du point de vue de l'élève, un certain nombre de «trous» constituant autant d'éléments non maîtrisés (Johsua \& Félix, 2002), l'enseignante délimite clairement au cours des échanges, ceux qui sont autorisés à la discussion dans la classe.

Par exemple, alors que les élèves cherchent en classe dédoublée à regrouper selon leurs propriétés différentes sources primaires et secondaires (min. 12), elle renvoie à plus tard la question d'un élève témoignant d'une conception de la

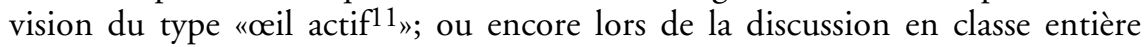
sur les objets diffusants, elle exclut une question sur les miroirs, alors que les commentaires du programme mentionnent que si l'occasion se présente, il est possible de différencier la diffusion de la réflexion. De manière plus délibérée, quand il s'agit de modéliser en classe dédoublée le phénomène de diffusion, elle affirme de manière dogmatique aux élèves qui contestent quasi-unanimement la propriété d'une source secondaire: «je vous dis moi, que la balle est une source de lumière» (min. 8). Cet élément - institutionnalisé de fait - ne constitue plus un objet de raisonnement mais un point d'appui, introduit pour raisonner dans le milieu. Elle présente ensuite (min. 13) une expérience dans laquelle elle va éclairer une balle blanche en lumière rouge, et demande aux élèves d'émettre des hypothèses sur ce qu'ils vont observer en regardant la lampe, puis en regardant la balle (min. 13 à 15). Cette injonction pointe les traits pertinents que les élèves devront observer dans la situation expérimentale à venir (min. 16). La professeure rend ainsi peu à peu possible le raisonnement qu'elle conduira ultérieurement avec les élèves (min. 22) quand ceux-ci auront formalisé leurs observations sur la fiche: si la balle blanche, source lumineuse, est vue rouge lorsqu'elle est éclairée par une lumière rouge, c'est qu'elle réémet la lumière qu'elle reçoit. Ce raisonnement est ainsi préparé par les échanges qui précèdent l'expérimentation réalisée devant les élèves. Cette modalité de travail est systématique parce que "on ne fait pas une manipulation pour manipuler, il y a un objectif... il ne faut pas se lancer tête baissée» (entretien ante). Elle est prolongée à chaque occasion par une mise en relief tout aussi systématique des éléments importants, que ce 
soit au cours de l'expérience, au cours des échanges qui suivent le travail individuel de formalisation des observations et des interprétations, ou encore lors des phases d'institutionnalisation.

En délimitant le milieu et en indiquant les traits pertinents pour raisonner, en soulignant les points significatifs, l'enseignante "essaie d'aller à l'essentiel ${ }^{12}$ » (entretien ante). On peut penser que c'est une manière pour elle de concilier ses ambitions vis-à-vis des savoirs avec les caractéristiques des élèves pour rendre possible et productive une démarche collective de type maïeutique. D'ailleurs, elle «ne le ferait pas dans une classe normale» (entretien ante).

Toutefois, cette stratégie, mise en œuvre en classe dédoublée et en classe entière, donne lieu dans chacun des cas à une construction de la référence différente, co-construite dans la classe dédoublée, davantage prise en charge par l'enseignante en classe entière.

\section{Mise en œuvre différenciée de la stratégie précédente en classe dédoublée ou entière \\ Co-construction de la référence en classe dédoublée}

Le bon fonctionnement de la démarche de type maïeutique suppose à la fois que les élèves participent aux échanges et aient des choses à apporter susceptibles de faire avancer le savoir.

Pour ce qui est de la participation aux échanges, on peut constater que l'enseignante prend le temps en classe dédoublée de construire une réelle adhésion à la tâche. Par exemple, avant de travailler sur la question "pourquoi voit-on la balle si elle ne produit pas de lumière ?», elle demande aux élèves de classer en argumentant quatre sources de lumière (soleil, lampe, neige et balle de ping-pong). Elle cherche toujours à ouvrir le débat et relance régulièrement les échanges par des interventions sous forme de questions: ainsi, alors qu'un élève lui avait proposé un classement pertinent correspondant aux sources primaires et secondaires qu'elle aurait pu valider pour avancer, elle a sollicité deux autres élèves qui ont fait valoir d'autres arguments. On est dans une communication de type dialogique au cours de laquelle différents points de vue sont présentés et analysés (Mortimer \& Scott, 2003), ce qui favorise une implication épistémique des élèves. D'ailleurs, en réponse aux sollicitations dont ils font l'objet, la plupart d'entre eux interviennent en faisant des phrases construites (par exemple durant la min. 24: Ka: «non, il faut qu'il y ait aucun obstacle»; Ra: «il y a la lumière qui tape, qui va dans toute la salle, et tous les objets qu' elle a atteint, on les voit, grâce à la lumière»; Sa: «ils ont la même couleur, c'est pour ça que ça fait ça»), et interagissent souvent les uns avec les autres. On est dans une réelle co-construction de la référence par la majorité des élèves de la classe dédoublée et par l'enseignante. Celle-ci adopte une posture de mise en débat des traits pertinents du milieu (Schubauer-Léoni, 2008), susceptible de favoriser une attitude de secondarisation (cf. extrait 3, voir plus loin).

Dans la dynamique interactive de construction de la référence, si les élèves 
contribuent à faire avancer les savoirs, l'enseignante a cependant du mal à obtenir d'eux la conclusion qu'elle espère à l'issue des échanges lors des discussions qu'elle suscite. Elle est contrainte d'y mettre elle-même un terme en formulant la réponse. C'est le cas par exemple dans l'extrait 2 au cours duquel les élèves ont du mal à abandonner leur modèle spontané de la vision et réfutent quasi-unanimement la possibilité de la réémission de lumière par une source secondaire.

Extrait 2. Nécessité pour l'enseignante de conclure elle-même les échanges (classe dédoublée, minute 25). Cet extrait clôture les échanges dont une partie est présentée dans l'extrait 1.

P.: $\quad$ Donc moi je vous dis que la balle envoie de la lumière dans vos yeux. C'est ça qui est écrit. C'est possible ça?

Ho: Oui // Ka: Non // Mo: Non // Ho: Si!

Ka: Non, c'est pas possible

Ho: Je sais pas!

Ka: $\quad$ Parce que la boule, la balle, elle produit pas de lumière

$P: \quad$ Est-ce qu'elle en reçoit de la lumière?

Elèves: Oui

P: $\quad$ Est-ce qu'elle peut en renvoyer?

Elèves: Non

P: Non?

Ho: Si l/ Ka: Nooon, ça éclaire juste la balle, ça renvoie pas.

$P$ : En fait, c'est ce qu'il se passe... [Et l'enseignante explique le phénomène]

Cette forme d'institutionnalisation que le dogme constructiviste pourrait conduire à considérer comme prématurée, peut être envisagée dans sa fonction chronogénétique comme le discute Forget (2008). En tout cas, cette façon de faire ne semble pas nuire aux acquisitions (au moins à court terme), comme l'a montré l'entretien collectif peu de temps après la fin de séance avec des élèves « représentatifs» de la classe (extrait 3).

Extrait 3. Echanges entre élèves lors de l'entretien collectif, à propos de l'expérience réalisée en classe dédoublée

Ho: bon on a appris qu'il y avait deux sortes de lumières, qu'il y avait des lumières primaires et des lumières secondaires, les lumières primaires, c'est eux-mêmes qui fabriquent leur propre lumière, et en fait les secondaires, on envoie une quantité de lumière et après elle rejette, elle émet une autre partie, comme ça on peut la voir.

$\mathrm{Ka}: \quad$ elle diffuse, oué elle reçoit après elle émet

Ka: $\quad$ elle a mis un filtre rouge sur la lampe 
Sy: $\quad$ pour montrer que la balle elle prenait la couleur du filtre

Ka: pas obligé

$\mathrm{Ka}: \quad$ c'est pas obligé de prendre la couleur du filtre, elle peut prendre la couleur de la lampe, par exemple blanche si la lampe est blanche, on verra la balle blanc, si la lampe elle est verte, on va voir vert, si elle est rouge on va voir rouge, c'est pas obligé que ce soit un filtre

\section{Prise en charge par l'enseignante de la construction de la référence en classe entière}

Si la structure des activités est identique à celle qui est proposée en classe dédoublée, la durée des différentes phases est systématiquement réduite en classe entière: par exemple dans les épisodes analysés, l'installation du milieu dure deux minutes en classe entière contre quatre à cinq minutes en classe dédoublée, la phase de réflexion collective dure deux minutes contre sept, la phase d'institutionnalisation dure une minute contre trois en classe dédoublée, etc. Ce n'est pas la moindre complexité des concepts en jeu qui explique cette accélération chronogénétique. Les deux épisodes sont en effet relatifs au phénomène de diffusion. C'est la forme prise par la direction de l'étude qui est très différente. En classe entière, l'enseignante se donne beaucoup moins de temps pour installer puis construire avec les élèves les éléments constitutifs du milieu d'étude. Elle réduit les questions posées, elle rectifie rapidement les réponses lorsque ce ne sont pas celles qu'elle attend, elle est plus directive dans ses formulations qui n'ont plus pour objectif de relancer le débat comme en demi-classe mais qui visent à le fermer pour avancer, et c'est une attitude argumentée:

à partir du moment où ils sont en classe entière, et c'est pas que sur cette classe, je suis beaucoup plus obligée de prendre la classe en main et d'être dirigiste. Si je laisse s'instaurer les mêmes débats [qu'en demi-classe], très rapidement je ne tiens plus la classe, voilà, donc oui, ça c'est systématique (entretien post).

Aussi, même si la démarche maïeutique subsiste en classe entière, elle devient plus formelle (extrait 4), et les propos des élèves sont parfois «surexploités» (extrait 5).

Extrait 4. Prise en charge par l'enseignante de la construction de la référence (classe entière, minutes 40 à 41 ).

P: $\quad$ Qu'est ce qu'il se passe avec mon écran blanc là? [Il s'agit d'un écran blanc qui a une fonction diffusante dans l'expérience proposée].

El: $\quad$ Rien

El: $\quad Y$ renvoie

El: $\quad$ Il donne la lumière

P: $\quad$ C'est une source primaire ou secondaire?

Elèves: (les uns) Primaire! - (les autres) Secondaire!

P: $\quad$ SECONDAIRE! Alors rappelez moi comment fonctionne une source 


$\begin{array}{ll}\text { Mo: } & \text { elle reçoit de la lumière } \\ P: & \text { Elle reçoit de la lumière et elle en diffuse... } \\ E l: & \text { elle diffuuse! } \\ K a: & \text { elle émet! } \\ P: & \text { elle en diffuse une partie ! Et donc si je place l'objet dans la lumière que } \\ & \text { diffuse mon écran blanc, Qu'est ce qui va se passer? } \\ E l: & \text { On voit de la lumière rouge } \\ P: & \text { On voit pas de lumière rouge, c'est l'objet qui est rouge... } \\ E l: & \text { Elle [la balle] va pas devenir rouge! } \\ P: & \text { Bon on est d'accord? Je peux arriver à récupérer la lumière de la } \\ & \text { lampe alors que vous ne voyez pas directement la lampe et j'ai utilisé } \\ & \text { mon écran blanc comme une source secondaire qui va me permettre } \\ & \text { d'éclairer mon objet. }\end{array}$

Extrait 5. Surexploitation conclusive (classe entière, minute 38).

Mé: Ça [la lumière] occupe toute la pièce.

P: $\quad$ Comment ça se fait qu'elle va dans toute la pièce?

Mé: ben parce que...

Mo: $\quad$ elle réfléchit.

Mé: $\quad$ elle se diffuse.

P: $\quad$ Ab elle est diffusée effectivement

Sa: $\quad$ partout dans la pièce

P: $\quad$ par les objets qui nous entourent (respiration). Elle est diffusée par les objets qui nous entourent

Comme on peut le voir dans ces deux extraits, les élèves participent a minima, voire plus du tout. Par exemple à la minute 44, et contrairement à ce qu'il se passe en classe dédoublée, la participation des élèves, en réponse aux sollicitations de l'enseignante, se limite à «non !» et «inutile». Elle est même à un moment contrainte d'avancer seule, attendant intervention après intervention une réponse des élèves qui ne vient plus (min. 42): P: on vient de voir que notre écran blanc, diffuse la lumière, donc il renvoie ... Amelle! ... il renvoie ... Houssem assis, je t'assure que tu seras beaucoup mieux ... il renvoie... L'écran blanc renvoie la lumière... Issue de la source!

On peut donc dire qu'en classe entière, l'enseignante prend presque totalement à sa charge la construction de la référence dans la classe, les élèves échangeant peu entre eux et font souvent des réponses minimales: elle adopte en effet une posture en «surplomb» dans laquelle "elle garde le monopole du rapport aux objets» et elle statue «sur la pertinence/non pertinence des rapports aux objets de la leçon» (Schubauer-Leoni, 2008, p. 77). Parallèlement, l'ambiance se dégrade, 
et les rappels à l'ordre sont beaucoup plus nombreux qu'en classe dédoublée (par exemple six rappels à l'ordre lors de la minute 44 contre seulement quatre dans la totalité de l'heure en classe dédoublée). S'ils sont faits de manière ferme, ils ne sont jamais agressifs, comme on peut le voir dans l'extrait précédent minute 42, et ils pourraient être beaucoup plus nombreux. Cette attitude autorise probablement le maintien d'une relation didactique minimale avec les quelques élèves (surtout des garçons) qui, à tour de rôle s'engagent momentanément dans la tâche et lui permettent d'avancer dans la séance, d'autant que le cours n'est pas réellement perturbé par du bruit ou des altercations.

\section{Conclusion}

Après avoir observé et analysé ces deux séances en physique se déroulant en classe dédoublée et en classe entière dans un collège en milieu difficile, il nous semble possible de considérer que les pratiques de cette enseignante relèvent d'un genre «dominant» (au sens de Butlen, Peltier-Barbier, \& Pezard, 2002, p. 47). Celui-ci est en effet caractérisé par l'utilisation de scénarios avec résolution de problèmes, du temps laissé à la recherche, le souci de ne pas abaisser les exigences, l'alternance de phases individuelles et collectives, l'institutionnalisation en phases collectives, notamment après un travail individuel, des réinvestissements contextualisés et décontextualisés, un étayage important dans les phases de formulation, le souci de respecter les exigences et le temps institutionnel, des rappels à l'ordre individuels adaptés aux manifestations des élèves.

Nous avons observé que cette logique fonctionne si les élèves sont partenaires de l'action de l'enseignant, s'ils sont engagés dans les tâches proposées. On a pu voir que sous certaines conditions (identifier clairement ce quel est l'enjeu de savoir, se centrer sur l'essentiel et le souligner fortement, amener la conclusion pour couper court à des échanges improductifs, privilégier l'oral), cela est possible. Ce partenariat, cette co-construction de la référence dans la classe permet de résoudre une partie des paradoxes ou des dilemmes dans lesquels se débattent les enseignants en milieu difficile que nous évoquions précédemment. En particulier, l'engagement dont les élèves font preuve n'est pas conditionné par une réussite immédiate, ni par un sur-ajustement didactique au niveau des savoirs.

Toutefois, cette situation est beaucoup plus difficile à obtenir lorsque les élèves sont en classe entière: ils collaborent dans ce cas à tour de rôle, de manière ponctuelle et hétérogène. Malgré des interventions réitérées, l'enseignante n'arrive plus à créer les conditions de co-construction d'un milieu didactique «pertinent» relativement au savoir visé, elle prend en charge presque seule la production de la référence, se positionnant «en surplomb» (Schubauer-Leoni, 2008) pour faire avancer le savoir. Cette stratégie s'accompagne d'un ensemble de techniques visant dans un même temps à gérer la discipline afin de tenter de ramener les élèves dans la tâche. Dans les phases de formulation, les élèves participant peu 
et l'enseignante apportant beaucoup, ces deux attitudes se nourrissant l'une de l'autre, la chronogenèse s'accélère. Une des conséquences est qu'une partie de l'activité didactique se trouve alors empêchée tant au niveau des élèves que de l'enseignante (Monnier \& Amade-Escot, 2009, à paraître).

Outre la nécessité d'un partenariat élèves-enseignant dans la construction de la référence en classe, la comparaison de ces deux séances fait donc apparaître l'intérêt qu'il y a pour l'obtenir en milieu difficile, à travailler avec des groupes de taille réduite (voire ici très réduite puisque la classe dédoublée comporte huit à neuf élèves). Si on peut comprendre que l'enseignante ait alors un temps suffisant pour faire s'exprimer et mettre en débat les différents points de vue individuels, il resterait à voir, en comparant avec d'autres groupes, si au-delà de la taille réduite, il n'y a pas, pour expliquer ce qu'on a pu observer en classe dédoublée, des conditions particulières supplémentaires qui auraient été réunies, par exemple l'existence d'une majorité de garçons certes brouillons mais décidés à occuper le devant de la scène dans les interactions, ou une construction de la référence peu contrainte par des savoirs scolaires antérieurs puisqu'il s'agit des premiers contacts avec l'optique, ou encore une expertise professionnelle de l'enseignante dans la conduite mésogénétique en milieu difficile.

Enfin, au-delà de ce contexte particulier, certaines des conclusions précédentes nous paraissent transposables à des milieux plus ordinaires dans lesquels les phénomènes observés ici apparaissent aussi, même si c'est de manière moins tranchée. En effet, le positionnement de l'enseignant en surplomb existe aussi dans les classes ordinaires en parallèle avec une difficulté à enrôler les élèves (Blatchford, Bassett $\&$ et Brown, 2008) ou même dans certaines très bonnes classes (Venturini, Calmettes, Amade-Escot \& Terrisse, 2007), même si cela ne se solde pas par du bruit ou de l'agitation.

\section{Notes}

1 D'après les programmes officiels, la démarche «d'investigation» comporte sept «moments essentiels»: choix d'une situation problème par le professeur, appropriation du problème par les élèves, formulation d'hypothèses et de protocoles expérimentaux, investigation et résolution du problème conduite par les élèves, échanges argumentés autour des propositions élaborées, acquisition et structuration des connaissances, opérationnalisation des connaissances.

2 Dans cet article, les pratiques de références sont liées à l'usage quotidien des savoirs de l'optique, comme préconisé dans les programmes.

3 Celle qui résulte du cheminement dialectique sur le continuum évoqué précédemment.

4 Ce terme ne réfere bien sûr pas seulement à des objets matériels.

5 Ces deux dimensions du milieu renvoient au continuum «interne-externe» évoqué précédemment

6 Etymologiquement, la mésogénèse renvoie à la genèse du milieu. Le contenu des transactions didactiques évolue en effet régulièrement dans le temps, au fur et à mesure de l'avancée des savoirs, notamment sous l'influence des régulations de l'enseignant et des apports des élèves. Etudier la mésogenèse c'est donc «étudier comment le contenu de l'interaction en continu se trouve co-élaboré par le professeur et les élèves» (Sensevy, 2007, p. 30).

7 Les tâches didactiques ont la particularité d'être généralement coopératives, leur accom- 
plissement supposant l'engagement de l'enseignant et de l'élève, dans une interaction (Chevallard, 1999). Chacun d'entre eux, tient dans cette interaction un rôle particulier vis-à-vis du savoir, son «topos» (Chevallard, 1992), qui lui appartient et engage sa responsabilité. L'évolution entre les acteurs, du partage des responsabilités relatives à l'avancée du savoir au cours du temps, constitue la topogénèse.

8 Dans ces tableaux, nous avons repris en italique les questions figurant sur les fiches-élèves.

9 Al-Azhem est un scientifique arabe ayant entre autre «modélisé» aux alentours de l'an 1000 , le principe de la vision des objets.

10 Selon les programmes (MEN, 2007), les élèves doivent savoir que «le Soleil, les étoiles et les lampes sont des sources primaires; la lune, les planètes, les objets éclairés sont des objets diffusants. Pour voir un objet, il faut que l'œil en reçoive de la lumière» (p. 120).

11 Il s'agit d'une conception assez marginale selon laquelle l'œil émet des «rayons» permettant de «percevoir» l'objet éclairé.

12 "L'essentiel» pour l'enseignante, correspond strictement aux exigences des programmes, comme on peut le voir en comparant ces dernières décrites dans la note 10, aux enjeux didactiques des différents épisodes rapportés dans les tableaux 1 et 2 ou encore dans la section Résultats. "Aller à l'essentiel», toujours pour l'enseignante, c'est éliminer des échanges tout ce qui est inutile pour parvenir à ces conclusions, comme le montre l'analyse précédente.

\section{Références bibliographiques}

Amade-Escot, C. (2002). Etude du travail de l'enseignant d'éducation physique dans la classe: contribution des recherches didactiques à l'analyse des pratiques effectives. In J. F. Marcel (Ed.), Les Sciences de l'Education: des recherches, une discipline (pp. 53-78). Paris: L'Harmattan, Collection Savoir et Formation.

Amade-Escot, C. \& Venturini, P. (2009). Le milieu didactique: d'une étude empirique en contexte difficile à une réflexion sur le concept. Education et Didactique, 3 (1) (à paraître).

Andersson, B. \& Karrqvist, C. (1983). How swedish pupils; aged 12-15 years, understand light and its properties. European Journal of Science Education, 5 (4), 387-402.

Bautier, E. \& Goigoux, R. (2004). Difficultés d'apprentissage, processus de secondarisation et pratiques enseignantes: une hypothèse relationnelle. Revue Française de Pédagogie, 148, 89-100.

Blatchford, P., Bassett, P. \& Brown, P. (2008). Do low attaining and younger students benefit most from small classes ? Results from a systematic observation study of class size effects on pupil classroom engagement and teacher pupil interaction. Communication présentée lors de l'American Educational Research Association Annual Meeting: Research on Schools, Neighborhoods, and Communities: Toward Civic Responsibility. New York: (24-28 mars). Consulté le 20 avril 2009 dans http://www.classsizeresearch.org.uk/aera\%2008\%20paper.pdf

Brousseau, G. (1986). Fondements et méthodes en didactique des mathématiques. Recherches en Didactiques des Mathématiques, 7 (2), 33-115.

Brousseau, G. (1990). Le contrat didactique: le milieu. Recherches en Didactique des Mathématiques, 9 (3), 309-336.

Butlen, D., Peltier-Barbier, M.-L. \& Pezard, M. (2002). Nommés en REP ? Comment fontils? Pratiques de professeurs d'école enseignant les mathématiques en REP: contradiction et cohérence. Revue Française de Pédagogie, 140, 41-52.

Chevallard, Y. (1991) La transposition didactique. Du savoir savant au savoir enseigné (2e édition revue et augmentée, en coll. avec M-A. Joshua, 1e édition 1985). Grenoble: La Pensée Sauvage.

Chevallard, Y (1992). Concepts fondamentaux de la didactique: perspective apportée par une 
approche anthropologique. Recherches en Didactique des Mathématiques, 12 (1), 73-112.

Chevallard, Y. (1999). L'analyse des pratiques enseignantes en théorie anthropologique du didactique. Recherche en Didactique des Mathématiques, 19 (2), 222-265.

Forget, A. (2008). L'institutionnalisation en classe de français: effets sur l'identité conceptuelle et empirique du concept originel. Dossiers des Sciences de l'éducation, 20, 75-88.

Johsua, S. (2002). Spécificités disciplinaires, spécificités didactiques, vers une didactique comparée. In P. Venturini, C. Amade-Escot \& A. Terrisse (Éd.), Étude des pratiques effectives: l'approche des didactiques (pp. 17-24). Grenoble: La Pensée Sauvage.

Johsua, S. \& Félix, C. (2002). Le travail des élèves à la maison: une analyse didactique en termes de milieu pour l'étude. Revue Française de Pédagogie, 141, 89-97.

Leutenegger, F. (2003). Étude des interactions didactiques en classe de mathématiques: un prototype méthodologique. Bulletin de Psychologie, 56 (4), 559-571.

MEN, (2007). Programmes de sciences physiques, classe de cinquième. Bulletin Officiel de l'Éducation Nationale, hors série 6 (volume 2 du 19 avril 2007).

Mercier, A., Schubauer-Leoni, M. \& Sensevy, G. (2002). Vers une didactique comparée. Revue Française de Pédagogie, 141, 5-16.

Monnier, N. \& Amade-Escot, C. (2009). L'activité didactique empêchée: un outil d'intelligibilité de la pratique enseignante en milieu difficile. Revue Française de Pédagogie, 167, (à paraître).

Mortimer, E. \& Scott, P. (2003). Meaning making in secondary science classrooms. Maidenhaed: Open University Press.

Peltier-Barbier, M.-L. (2004). Dur d'enseigner en ZEP. Dur pour les élèves, Dur pour les enseignants. Grenoble: Éditions La Pensée sauvage.

Rochex, J.-Y. (1997). Les ZEP un bilan décevant. In J.-P. Terrail (Éd.), La scolarisation de la France: Critique de l'état des lieux (pp. 123-140). Paris: La Dispute.

Rochex, J.-Y., \& Kherroubi, M. (2004). La recherche en éducation et les ZEP en France 2. Apprentissage et exercice professionnel en ZEP: résultats, analyse, interprétations (note de synthèse). Revue Française de Pédagogie, 146, 115-190.

Schubauer-Leoni M.-L. (2008). La construction de la référence dans l'action conjointe professeur-élèves. In N. Wallian, M.-P. Poggi \& M. Musard (Éd.) Co-construire des savoirs: les métiers de l'intervention dans les APSA (pp. 67-86). Besançon: Presses universitaires de Franche Comté.

Schubauer-Leoni, M.-L. \& Leutenegger, F. (2005). Une relecture des phénomènes transpositifs à la lumière de la didactique comparée. Revue Suisse des Sciences de l'Éducation, 27 (3), 407-427.

Selley, N. J. (1996). Towards a phenomenography of light and vision, International Journal of Science Education, 18 (7), 837-846.

Sensevy, G. (2007) . Des catégories pour décrire et comprendre l'action didactique. In G. Sensevy \& A. Mercier (Ed.), Agir ensemble: l'action didactique conjointe du professeur et des élèves (pp. 13-49). Rennes: PUR.

Sensevy, G. \& Mercier, A. (2007) (Dir.). Agir ensemble: l'action didactique conjointe du professeur et des élèves. Rennes: Presses Universitaires de Rennes.

Sensevy, G. \& Mercier, A. (2007). Agir ensemble : l'action didactique conjointe. In G. Sensevy \& A. Mercier, (2007) (dir.), Agir ensemble : l'action didactique conjointe du professeur et des élèves (pp. 187-211). Rennes : Presses Universitaires de Rennes.

Sensevy, G., Mercier, A. \& Schubauer-Leoni, M.-L. (2000). Vers un modèle de l'action didactique du professeur à propos de la course à 20. Recherches en Didactique des Mathématiques, 20 (3), 263-304.

Tiberghien, A. (1983). Revue critique sur les recherches visant à élucider le sens de la notion de lumière pour les élèves de 10 à 16 ans. In Atelier international d'été: recherche en didactique de la physique (pp.125-133). Paris, La Londe des Maures: CNRS.

Van Zanten, A. (2001). L'école de la périphérie. Scolarité et ségrégation en banlieue. Paris: 
Presses Universitaires de France.

Venturini, P., Calmettes, B., Amade-Escot, C. \& Terrisse, A. (2007). Analyse didactique des pratiques d'enseignement de la physique d'une professeure expérimentée. Aster, 45, 211-234.

Mots-clés: Co-construction de la référence, milieu difficile, théorie de l'action conjointe, didactique de la physique, gestion de classe

\section{Physikunterricht an Brennpunktschulen: Nachbau einer Referenz mit der halben und der ganzen Klasse Résumé}

\section{Zusammenfassung}

Um gewöhnliche Unterrichtspraktiken verständlicher zu machen, vergleicht dieser Beitrag die Methode, wie eine Physiklehrerin in einer Brennpunktschule eine Referenz nachbildet, einerseits mit der halben Klasse, andererseits mit der ganzen Klasse. Der Artikel zeigt, dass es unter bestimmten Bedingungen mit der Hälfte der Klasse eher möglich ist, eine effektive Partnerschaft aufzubauen, um den Lehrstoff zu vermitteln. Es wurde ebenfalls festgestellt, dass dieselben Methoden, angewandt in der ganzen Klasse, weniger wirksam sind.

Schlagworte: Ko-konstruktion von Referenzen, soziale Benachteiligung, kooperatives Handeln, Physikdidaktik, Klassenführung

\section{Insegnare la fisica in contesti difficili: co-costruzione della referenza in una classe dimezzata $e$ in una classe intera}

\section{Riassunto}

Allo scopo di capire meglio le pratiche d'insegnamento abituali, questo articolo confronta il modo in cui una insegnante di fisica co-costruisce con i suoi allievi la referenza in un classe dimezzata e una classe intera, in una zona di educazione prioritaria. Si tratta di mostrare come in una classe dimezzata sia possibile stabilire un partenariato effettivo con gli allievi per fare avanzare, ad alcune condizioni, le conoscenze. Si mostra anche che le strategie messe in atto sono assai meno operanti in una classe intera, dal momento in cui la collaborazione risulta più aleatoria; questo spinge l'insegnante ad adottare una postura di "surplomb".

Parole chiave: Co-costruzione della referenza, contesti difficili, teoria dell'azione congiunta, didattica della fisica, gestione della classe 


\section{Teaching physics in a deprived urban area: co-construction of the reference with a half class group and the whole class group.}

\section{Abstract}

To better understand ordinary teaching practices, this article compares the way a physics teacher co-constructed reference with her students working with a half class group and with the whole class group. We observed that when the teacher works in a half class group, she succeeds conditionally in building an effective partnership with students for elaborating knowledge. We also observed that the strategies she uses are less effective in the whole class, collaboration being more uncertain. This leads the teacher to get an «overhanging» posture.

Key words: Reference co-construction, deprived area, didactic joint action, physics didactics 
